\title{
Blind Channel Estimation for Cyclic-Prefixed Single-Carrier Systems by Exploiting Real Symbol Characteristics
}

\author{
Feifei Gao, Student Member, IEEE, Arumugam Nallanathan, Senior Member, IEEE, and \\ Chintha Tellambura, Senior Member, IEEE
}

\begin{abstract}
In this paper, we consider blind channel estimation for cyclic-prefixed single-carrier (CP-SC) systems via secondorder statistics only. By exploiting the existence of real symbols in the transmitted data block, we develop a simple technique for blind channel estimation that does not need redundant precoding or virtual carriers. Hence, the proposed algorithm provides bandwidth efficiency. A side benefit of the proposed algorithm is that the phase ambiguity, which is an inherent problem in traditional blind channel estimation, is reduced to sign ambiguity only. This side benefit facilitates signal detection in digital communications where symbols are usually taken from a finite alphabet. Although the proposed method is mainly developed for channel estimation in single-input-single-output CP-SC systems, it can also be applied straightforwardly to single-input-multiple-output systems (either with CP-SC or other kinds) and multiple-input-multiple-output systems whenever real symbols exist or are deliberately inserted in the transmission.
\end{abstract}

Index Terms-Blind channel estimation, cyclic prefixed single carrier (CP-SC), IEEE 802.11a, orthogonal frequency-division multiplexing (OFDM), subspace methods, wireless communications, Cramér-Rao bound (CRB).

\section{INTRODUCTION}

C YCLIC-PREFIXED single-carrier (CP-SC) communication with frequency-domain equalization (SC-FDE) [1]-[4] has recently received more attention since it has nearly all the advantages of orthogonal frequency-division multiplexing $(\mathrm{OFDM})^{1}$ but offers significant performance improvements over OFDM in certain environments. SC-FDE delivers performance similar to OFDM with essentially the same overall complexity [1]. In addition, since the peak-to-average power ratio (PAPR) in SC-FDE is much smaller, more powerefficient transmitters can be used. Several training-sequence-

Manuscript received January 4, 2006; revised July 16, 2006, October 27, 2006, and December 5, 2006. The review of this paper was coordinated by Prof. R. Heath.

F. Gao and A. Nallanathan are with the Department of Electrical and Computer Engineering, National University of Singapore, Singapore 119260 (e-mail: feifeigao@nus.edu.sg; elena@nus.edu.sg).

C. Tellambura is with the Department of Electrical and Computer Engineering, University of Alberta, Edmonton, AB T6G 2V4, Canada (e-mail: chintha@ece.ualberta.ca).

Digital Object Identifier 10.1109/TVT.2007.899945

${ }^{1}$ OFDM [5], [6] is a promising candidate for next-generation high-speed wireless multimedia communication systems. It has been used in European digital audio/video broadcasting [7], [8] and high-performance local-area network [9] and 802.11a wireless LAN standards [10]. based channel-estimation algorithms for CP-SC systems are developed in [3] and [4].

A promising blind-channel-estimation method, known as the subspace-based method, has been developed in [11]. In this method, the observation space is separated into signal and noise subspaces by applying eigenvalue decomposition on the covariance matrix of the received signals. By exploiting the inherent structure of the channel matrix, the channel vector can be estimated via the noise subspace up to a complex scalar ambiguity. For OFDM or CP-SC transmission, however, the subspace-based method cannot be applied directly since the channel matrix is square, and no dimension of the noise subspace exists.

In order to solve this problem, some redundancy must be introduced in each data block. For example, a linear-blockprecoding technique is carried on in [12], and virtual carriers are exploited in [13]. However, both these techniques sacrifice at least one symbol in each block in order to construct a tall channel matrix. A nonredundant linear-precoding method is developed in [14]. However, this method requires transmitted symbols on all time slots to be independent and have equal power, which may not be fulfilled in practical applications.

In this paper, we propose a real-symbol-combined blindchannel-estimation method for CP-SC transmissions, aiming to cope with the square channel matrix, to improve estimate accuracy, as well as to increase the bandwidth efficiency. The proposed method needs only one symbol in each data block to be real; thus, it loses only "half" of a symbol when a complex constellation is employed. Compared to [12] and [13], the proposed method provides higher bandwidth efficiency. Despite this "half" symbol sacrifice being an overhead, the following advantages encourage a study of the proposed method:

1) successful application of a subspace-based algorithm to the square channel matrix and a reduction on the data rate loss;

2) performance improvement by a thorough exploitation of the inner structure of the transmitted symbols;

3 ) reduction of the phase ambiguity to sign ambiguity;

4) easy extension to other systems.

This paper is organized as follows. Section II presents the system model of CP-SC transmission. Section III provides our proposed algorithm and several related theorems. Section IV presents the Cramér-Rao bound (CRB) of the proposed estimator. In Section V, we provide the simulation results illustrating 


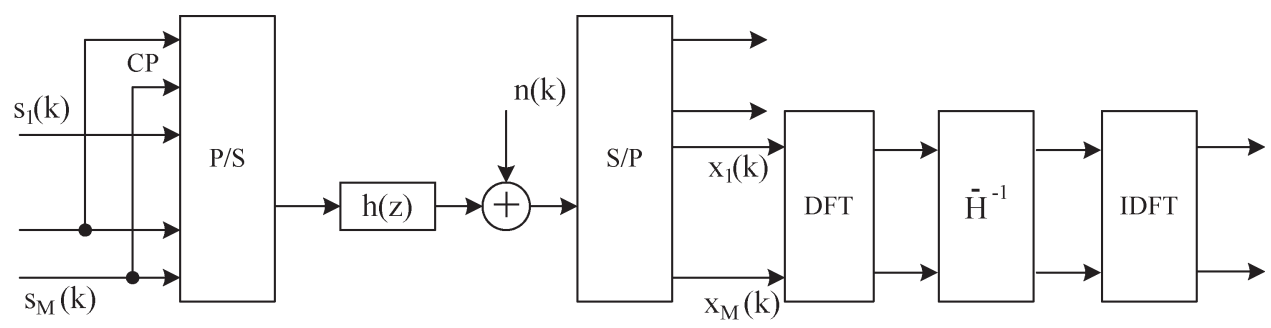

Fig. 1. Equivalent discrete baseband CP-SC system model.

the effectiveness of our algorithm. Finally, conclusions are made in Section VI, and proofs of theorems are given in the Appendices.

Notation: vectors and matrices are boldface small and capital letters; the transpose, complex conjugate, Hermitian, inverse, and pseudoinverse of matrix $\mathbf{A}$ are denoted by $\mathbf{A}^{\mathrm{T}}, \mathbf{A}^{*}, \mathbf{A}^{\mathrm{H}}$, $\mathbf{A}^{-1}$, and $\mathbf{A}^{\dagger}$, respectively; $\operatorname{tr}(\mathbf{A})$ and $\|\mathbf{A}\|_{\mathrm{F}}$ are the trace and the Frobenius norm of $\mathbf{A} ; \operatorname{diag}\{\mathbf{a}\}$ denotes a diagonal matrix with the diagonal element constructed from $\mathbf{a} ; \operatorname{Re}\{\mathbf{A}\}$ and $\operatorname{Im}\{\mathbf{A}\}$ represent the real and imaginary part of a matrix; $\otimes$ stands for the Kronecker product; $\mathbf{I}$ is the identity matrix; and $\mathrm{E}\{\cdot\}$ denotes the statistical expectation. The MATLAB notations for rows and columns are used. For example, $\mathbf{A}(m,:)$ represents the $m$ th row of the matrix $\mathbf{A}$, and $\mathbf{A}\left(m_{1}: m_{2},:\right)$ represents the submatrix obtained by extracting rows $m_{1}$ through $m_{2}$ from matrix $\mathbf{A}$, respectively.

\section{System Model For CP-SC TRANSMission}

Fig. 1 shows the baseband CP-SC system that we consider. The discrete channel response is represented by the $(L+1) \times 1$ complex vector $\mathbf{h}=\left[h_{0}, \ldots, h_{L}\right]^{\mathrm{T}}$. The $\mathrm{CP}$ is added in front of each transmitted block and is discarded at each received block. As long as the length of the $\mathrm{CP}$ is greater than or equal to $L$, the remaining signal at the receiver for the $k$ th block can be represented as

$$
\mathbf{x}(k)=\mathbf{H} \mathbf{s}(k)+\mathbf{n}(k)
$$

where $\mathbf{s}(k)$ is the $M \times 1$ time-domain transmitted symbol vector, $\mathbf{n}(k)$ is the $M \times 1$ vector of the white Gaussian noise terms with equivalent variance $\sigma^{2}, \mathbf{H}$ is the circulant channel matrix with its $(i, j)$ th entry, which is given by $\mathbf{h}_{M}((i-j) \bmod M)$, and the $M \times 1$ vector $\mathbf{h}_{M}$ is obtained by inserting $M-L-1$ zeros at the end of $\mathbf{h}$.

Define the $M \times M$ normalized discrete Fourier transform (DFT) matrix $\mathbf{F}$ with its $(q, p)$ th entry, which is given by $e^{-j 2 \pi(q-1)(p-1) / M} / \sqrt{M}$. The normalized DFT of the received signal vector is

$$
\mathbf{y}(k)=\mathbf{F} \mathbf{x}(k)=\overline{\mathbf{H}} \mathbf{F} \mathbf{s}(k)+\mathbf{F n}(k)
$$

where $\overline{\mathbf{H}}$ is a diagonal matrix with the form $\overline{\mathbf{H}}=$ $\operatorname{diag}\left\{\operatorname{DFT}\left(\mathbf{h}_{M}\right)\right\}$. Therefore, a one-tap equalization can be performed in the frequency domain and the symbols can be recovered by

$$
\mathbf{s}(k)=\mathbf{F}^{\mathrm{H}} \overline{\mathbf{H}}^{-1} \mathbf{y}(k)=\mathbf{F}^{\mathrm{H}} \overline{\mathbf{H}}^{-1} \mathbf{F} \mathbf{x}(k) .
$$

This is the so-called SC-FDE approach. As stated in [1], the SC-FDE approach has essentially the same performance and low complexity as an OFDM system but does not suffer from the PAPR problem. Therefore, the CP-SC system is a potential candidate for broadband wireless communications.

\section{Blind Channel Estimation By Exploiting REAL SYMBOLS}

\section{A. Model for Real-Symbol-Combined Systems}

For subspace-based blind channel estimation, the channel matrix in (1) and (2) must be a full-rank tall matrix, i.e., the number of rows is greater than the number of columns. Unfortunately, both $\mathbf{H}$ and $\overline{\mathbf{H}} \mathbf{F}$ are square matrices, and the subspace-based method cannot be applied directly. However, a tall channel matrix can be realized by assigning redundancy into $\mathbf{s}(k)$. Normally, at least one symbol redundancy should be added into each data block, as done in [12] and [13]. In this paper, we propose a new subspace-based blind channel estimation that requires only one real symbol in each data block. This real symbol can either be obtained by deliberate transmission, or it may already exist for certain systems, e.g., BPSK/pulse amplitude modulation (PAM) systems [15], [16].

For a generic discussion, we assume that there exist $m$ real symbols at fixed positions in each data block and that all other symbols are taken from arbitrary signal constellations. Without loss of generality, we assume that the first $m$ symbols of each block are BPSK/PAM symbols. After stacking the received signal and its complex conjugate in one vector, we get

$$
\breve{\mathbf{x}}(k)=\breve{\mathbf{H}} \breve{\mathbf{s}}(k)+\breve{\mathbf{n}}(k)
$$

where

$$
\begin{aligned}
\breve{\mathbf{x}}(k) & =\left[\mathbf{x}(k)^{\mathrm{T}}, \mathbf{x}(k)^{\mathrm{H}}\right]^{\mathrm{T}} \\
\breve{\mathbf{H}}(:, 1: m) & =\left[\mathbf{H}(:, 1: m)^{\mathrm{T}}, \mathbf{H}(:, 1: m)^{\mathrm{H}}\right]^{\mathrm{T}} \\
\breve{\mathbf{s}}(k) & =\left[\mathbf{s}(k)^{\mathrm{T}}, \mathbf{s}(k)(m+1: M)^{\mathrm{H}}\right]^{\mathrm{T}} \\
\breve{\mathbf{H}}(:, m+1: M) & =\left[\mathbf{H}(:, m+1: M)^{\mathrm{T}}, \mathbf{0}\right]^{\mathrm{T}} \\
\breve{\mathbf{n}}(k) & =\left[\mathbf{n}(k)^{\mathrm{T}}, \mathbf{n}(k)^{\mathrm{H}}\right]^{\mathrm{T}} \\
\breve{\mathbf{H}}(:, M+1: 2 M-m) & =\left[\mathbf{0}, \mathbf{H}(:, m+1: M)^{\mathrm{H}}\right]^{\mathrm{T}}
\end{aligned}
$$

and $\mathbf{0}$ is the $(M-m) \times M$ zero matrix.

Theorem 1: If there exists only one real symbol in each block, namely, $m=1$, the matrix $\mathbf{H}$ would be a $2 M \times(2 M-1)$ 
full-rank matrix if and only if the channel frequency response $h\left(e^{j \omega}\right) \triangleq \sum_{l=0}^{L} h_{l} e^{-j l \omega}$ has no nulls at $\omega_{M}=(2 \pi m / M), m=$ $0, \ldots, M-1$.

Proof: Let

$$
\tilde{\mathbf{H}}=\left[\begin{array}{cc}
\mathbf{H} & \mathbf{0} \\
\mathbf{0} & \mathbf{H}^{*}
\end{array}\right], \quad \tilde{\mathbf{H}}=\left[\begin{array}{cc}
\overline{\mathbf{H}} & \mathbf{0} \\
\mathbf{0} & \overline{\mathbf{H}}^{*}
\end{array}\right], \quad \tilde{\mathbf{F}}=\left[\begin{array}{cc}
\mathbf{F} & \mathbf{0} \\
\mathbf{0} & \mathbf{F}^{*}
\end{array}\right] .
$$

Matrix $\breve{\mathbf{H}}$ in (4) can be expressed as

$$
\breve{\mathbf{H}}=\tilde{\mathbf{H}} \mathbf{T}=\tilde{\mathbf{F}}^{\mathrm{H}} \tilde{\overline{\mathbf{H}}} \tilde{\mathbf{F}} \mathbf{T}
$$

where

$$
\mathbf{T}=\left[\begin{array}{ll}
\mathbf{I}_{M \times M} & \mathbf{0}_{M \times(M-1)} \\
1 & \mathbf{0}_{1 \times(2 M-2)} \\
\mathbf{0}_{(M-1) \times M} & \mathbf{I}_{(M-1) \times(M-1)}
\end{array}\right]
$$

is a $2 M \times(2 M-1)$ full-rank matrix. It follows that

$$
\operatorname{rank}\{\breve{\mathbf{H}}\}=\operatorname{rank}\{\tilde{\mathbf{F}} \breve{\mathbf{H}}\}=\operatorname{rank}\{\tilde{\overline{\mathbf{H}}} \tilde{\mathbf{F}} \mathbf{T}\}
$$

and

$$
\operatorname{rank}\{\tilde{\mathbf{F}} \mathbf{T}\}=\operatorname{rank}\{\mathbf{T}\}=2 M-1 .
$$

Sufficient Condition: If no channel null exists on the $M$ fast Fourier transform frequency points of $\mathbf{h}$, then

$$
\operatorname{rank}\{\tilde{\mathbf{H}} \tilde{\mathbf{F}} \mathbf{T}\}=\operatorname{rank}\{\tilde{\mathbf{F}} \mathbf{T}\}=2 M-1 .
$$

Therefore, $\breve{\mathbf{H}}$ is a full-rank matrix. The sufficient condition is proved.

Necessary Condition: This condition is proved by contradiction. Suppose there exists a channel null at $\omega=e^{j(2 \pi p / M)}$, $p \in\{0, \ldots, M-1\}$, while $\operatorname{rank}\{\breve{\mathbf{H}}\}$ still keeps $2 M-1$. Since $\overline{\mathbf{H}}(p+1, p+1)=0$, it follows that $\overline{\mathbf{H}}(p+1, p+1)^{*}=0$. Hence, two diagonal entries of $\tilde{\overline{\mathbf{H}}}$ are zeros. Therefore

$$
\operatorname{rank}\{\breve{\mathbf{H}}\}=\operatorname{rank}\{\tilde{\overline{\mathbf{H}}} \tilde{\mathbf{F}} \mathbf{T}\} \leq 2 M-2 .
$$

By contradiction, the necessary condition is proved.

Theorem 2: If there exists more than one real symbol in each block, namely, $m \geq 2$, a sufficient condition for matrix $\breve{\mathbf{H}}$ to be full-column rank is that the channel frequency response should have no nulls at $\omega_{m}, m=0, \ldots, M-1$.

The proof can be easily seen from the proof for Theorem 1 . However, for more than one real symbol, the necessary condition is affected by many factors, e.g., $M, m$, the position of real symbols, and the position of null carriers. Therefore, it is difficult to arrive at a general conclusion. Normally, the more the real symbols exist per block, the looser the necessary condition will be. One can see this from the full real-symbol case, where the necessary condition can be readily shown to be that the channel frequency response has no more than $M / 2$ zeros at $\omega_{m}, m=0, \ldots, M-1$.

Furthermore, since each element of $\mathbf{n}(k)$ is assumed to be a zero-mean white Gaussian complex circular variable with variance $\sigma^{2}$, the new noise vector $\breve{\mathbf{n}}(k)$ has the covariance

$$
\mathrm{E}\left\{\breve{\mathbf{n}}(k) \breve{\mathbf{n}}(k)^{\mathrm{H}}\right\}=\sigma^{2} \mathbf{I}_{2 M} .
$$

Therefore, by letting $m \geq 1$ symbols in each block be real, the new model (4) results in a tall channel matrix, such that the problem of channel estimation can be solved by subspace-based methods.

\section{B. Algorithm}

The signal covariance matrix can be expressed as

$$
\mathbf{R} \triangleq \mathrm{E}\left\{\breve{\mathbf{x}}(k) \breve{\mathbf{x}}(k)^{\mathrm{H}}\right\}=\breve{\mathbf{H}} \mathbf{R}_{\mathbf{s}} \breve{H}^{\mathrm{H}}+\sigma^{2} \mathbf{I}_{2 M}
$$

where $\mathbf{R}_{\mathbf{s}}=\mathrm{E}\left\{\breve{\mathbf{s}}(k) \breve{\mathbf{s}}(k)^{\mathrm{H}}\right\}$ is the source covariance matrix. If $\breve{\mathbf{H}}$ is of full column rank and the sources are not fully correlated, then $\mathbf{R}_{\mathrm{s}}$ is a nonsingular matrix, and $\mathbf{R}$ can be eigendecomposed as

$$
\mathbf{R}=\mathbf{E} \boldsymbol{\Lambda} \mathbf{E}^{\mathrm{H}}+\sigma^{2} \mathbf{G} \mathbf{G}^{\mathrm{H}}
$$

where the $(2 M-m) \times(2 M-m)$ diagonal matrix $\boldsymbol{\Lambda}$ contains the $2 M-m$ signal-subspace eigenvalues of $\mathbf{R}$, and the columns of the $2 M \times(2 M-m)$ matrix $\mathbf{E}$ contain the signalsubspace eigenvectors of $\mathbf{R}$. The $2 M \times m$ matrix $\mathbf{G}$ contains the noise-subspace eigenvectors of $\mathbf{R}$. We can show that

$$
\mathbf{G}^{\mathrm{H}} \breve{\mathbf{H}}(:, i)=0 \quad i=1, \ldots, 2 M-m .
$$

Define the $2 M \times 2(L+1)$ selection matrix

$$
\mathcal{J}_{i}= \begin{cases}{\left[\begin{array}{cc}
\mathbf{J}_{i} & \mathbf{0} \\
\mathbf{0} & \mathbf{J}_{i}
\end{array}\right],} & \text { for } 1 \leq i \leq m \\
{\left[\begin{array}{cc}
\mathbf{J}_{i} & \mathbf{0} \\
\mathbf{0} & \mathbf{0}
\end{array}\right],} & \text { for } m+1 \leq i \leq m \\
{\left[\begin{array}{cc}
\mathbf{0} & \mathbf{0} \\
\mathbf{0} & \mathbf{J}_{i-M+m}
\end{array}\right],} & \text { for } M+1 \leq i \leq 2 M-m\end{cases}
$$

where $\mathbf{J}_{i}$ is the $M \times(L+1)$ selection matrix, with its $(p, q)$ th entry given by

$$
\mathbf{J}_{i}(p, q)= \begin{cases}1, & \text { if }(p-q) \bmod M=i-1 \\ 0, & \text { otherwise }\end{cases}
$$

and let $\breve{\mathbf{h}}=\left[\mathbf{h}^{\mathrm{T}}, \mathbf{h}^{\mathrm{H}}\right]^{\mathrm{T}}$ denote the combination of both $\mathbf{h}$ and its conjugate. By proper rearrangement of the matrix and vector entries, it follows from (16) that

$$
\mathbf{G}^{\mathrm{H}} \mathcal{J}_{i} \breve{\mathbf{h}}=\mathbf{0} .
$$

From (19), the following equation can be obtained:

$$
\breve{\mathbf{h}}^{\mathrm{H}} \mathcal{J}_{i}^{\mathrm{H}} \mathbf{G G}^{\mathrm{H}} \mathcal{J}_{i} \breve{\mathbf{h}}=0 .
$$

Let us define the matrix

$$
\boldsymbol{\Phi}=\sum_{i=1}^{2 M-m} \mathcal{J}_{i}^{\mathrm{H}} \mathbf{G G}^{\mathrm{H}} \mathcal{J}_{i}=\sum_{i=1}^{2 M-m} \mathcal{J}_{i}^{\mathrm{H}} \mathbf{P}_{\mathbf{G}} \mathcal{J}_{i}
$$


with $\mathbf{P}_{\mathbf{G}}=\mathbf{G G}^{\mathrm{H}}$ representing the projection matrix onto the noise subspace. We can readily show that $\breve{\mathbf{h}}$ is in the null space of $\boldsymbol{\Phi}$. Therefore, the estimate of $\breve{h}$, which is denoted as $\mathbf{h}^{\prime}$, becomes

$$
\begin{aligned}
\mathbf{h}^{\prime} & =\arg \min _{\|\mathbf{w}\|=1} \mathbf{w}^{\mathrm{H}} \mathbf{\Phi} \mathbf{w} \\
& =\arg \min _{\|\mathbf{w}\|=1} \mathbf{w}^{\mathrm{H}}\left(\sum_{i=1}^{2 M-m} \mathcal{J}_{i}^{\mathrm{H}} \mathbf{P}_{\mathbf{G}} \mathcal{J}_{i}\right) \mathbf{w} .
\end{aligned}
$$

The unit-norm constraint on $\mathbf{w}$ ensures the generation of a nontrivial solution of $\mathbf{h}^{\prime}$.

An inherent problem of the subspace-based algorithm is that $\breve{\mathbf{h}}$ can only be estimated up to a complex scalar ambiguity, namely

$$
\mathbf{h}^{\prime}=\left[\begin{array}{l}
\mathbf{h}_{1}^{\prime} \\
\mathbf{h}_{2}^{\prime}
\end{array}\right]=\alpha \breve{\mathbf{h}}=\alpha\left[\begin{array}{c}
\mathbf{h} \\
\mathbf{h}^{*}
\end{array}\right]
$$

where $\alpha$ is an unknown complex scalar. By further combining $\mathbf{h}_{1}^{\prime}$ and $\mathbf{h}_{2}^{\prime}$, we obtain

$$
\ddot{\mathbf{h}}=\mathbf{h}_{1}^{\prime}+\mathbf{h}_{2}^{\prime *}=\alpha \mathbf{h}+\alpha^{*} \mathbf{h}=\operatorname{Re}\{\alpha\} \mathbf{h} .
$$

Note that the estimate $\ddot{\mathbf{h}}$ is a scale of $\mathbf{h}$ by a real factor. Therefore, the proposed conjugate-combined algorithm forms an important contribution by resolving "most" phase ambiguity. However, the sign ambiguity still exists since the real scalar $\alpha$ can be negative. Yet, this alleviation of phase ambiguity offers benefits in specific cases. For example, 1) if 3-PSK constellation [17] or another nonsymmetrical constellation with respect to origin is used, then no further training is needed, and 2) for other constellations symmetric about origin, e.g., QPSK, QAM, we can transmit unknown sequence of symbols that are obtained from constellations nonsymmetric about origin, e.g., all-positive unknown-symbol sequence, to resolve the ambiguity. Compared to the traditional way to resolve the ambiguity where training symbols must be sent, our method would save at least half of the information during the ambiguity-resolving process.

Some remarks are made here in order to help readers to distinguish the proposed method with the traditional subspacebased methods.

1) Traditionally, additional training symbols are sent to resolve channel-estimation ambiguity. The methods in [12] and [13] not only lose one symbol in each data block but also send additional training symbols to resolve this ambiguity. The number of training symbols should exceed a certain value to achieve sufficient accuracy. In comparison, our proposed method not only saves half symbol in each data block but also reduce this ambiguity to sign ambiguity only. Thus, it is an attractive blindchannel-estimation method.

2) For channel estimation in single-input-singleoutput, single-input-multiple-output, and multiple-inputmultiple-output transmissions, where the channel matrix is already tall, our proposed method can nevertheless be applied whenever there are real symbols. Since the proposed method factors in the real-symbol characteristics when estimating the channel response, it further improves the estimation performance. As the simulations show below, our proposed method outperforms the traditional subspace-based methods in this case.

3) For channel estimation where the channel matrix is already tall, the traditional subspace-based method can be successfully applied even when the transmitted symbols are all complex. However, the phase ambiguity cannot be removed even if we combine the received signals and its conjugate part.

4) Adding more real symbols in one data block is not recommended since this would increase the data rate loss. One real symbol is enough for applying the proposed subspace-based channel estimation. However, we suggest using the proposed method for the cases where real symbols already exist in transmissions [15], [16].

\section{Identifiability}

Identifiability is critical in assuring the correctness and the uniqueness of the estimation result for an algorithm. Traditional identifiability problem is discussed in [11], [13], [18], etc. However, it is also known that identifiability is a tough problem which may not be fully addressed occasionally. For most cases, only the sufficient conditions on the channel identification are provided.

Since we are developing a new way of channel estimation, we currently provide only the sufficient condition on identifiability when all the transmitted symbols are real.

Theorem 3: Assume that all symbols transmitted in one block are real. The sufficient conditions for channel identifiability are as follows:

1) Polynomial $h(z)=\sum_{l=0}^{L} h_{l} z^{-1}$ does not have a real root.

2) Polynomial $h(z)$ does not have conjugate roots.

3) $M$ is greater than $2 L+1$.

Proof: See Appendix A.

Note that the first two conditions would be satisfied with probability one since $\mathbf{h}$ is continuous random vector. The third condition is satisfied by many transmission schemes, e.g., in IEEE 802.11a [10], $M=4 L=64$.

Although we can only provide a partial answer to the identifiability issue, we will, in the later simulations, numerically check the channel identifiability. It will be seen that $\mathbf{h}$ can be uniquely obtained with high probability.

\section{Reduction of Memory Requirements}

Due to the special structure of matrix $\breve{\mathbf{H}}$, the noise subspace matrix $\mathbf{G}$ exhibits the following property.

Theorem 4: Divide $\mathbf{G}$ as $\mathbf{G}=\left[\mathbf{G}_{1}^{\mathrm{T}}, \mathbf{G}_{2}^{\mathrm{T}}\right]^{\mathrm{T}}$, where $\mathbf{G}_{1}$ and $\mathbf{G}_{2}$ are two $M \times m$ matrices, and let $\mathbf{G}=\left[\mathbf{G}_{2}^{\mathrm{H}}, \mathbf{G}_{1}^{\mathrm{H}}\right]^{\mathrm{T}}$. It follows that

$$
\mathbf{P}_{\mathbf{G}}=\mathbf{G G}^{\mathrm{H}}=\tilde{\mathbf{G}} \tilde{\mathbf{G}}^{\mathrm{H}} .
$$

See the proof in Appendix B. 
Consequently, the following property holds:

$$
\begin{aligned}
& \mathbf{G}_{1} \mathbf{G}_{1}^{\mathrm{H}}=\left(\mathbf{G}_{2} \mathbf{G}_{2}^{\mathrm{H}}\right)^{*} \\
& \mathbf{G}_{1} \mathbf{G}_{2}^{\mathrm{H}}=\left(\mathbf{G}_{2} \mathbf{G}_{1}^{\mathrm{H}}\right)^{*} .
\end{aligned}
$$

Since both $\mathbf{G}$ and $\tilde{\mathbf{G}}$ are orthonormal matrices, there must exist an $m \times m$ unitary matrix $\mathbf{U}$, such that

$$
\mathbf{G}=\tilde{\mathbf{G}} \mathbf{U} .
$$

One can further obtain from (27) that $\left\|\mathbf{G}_{1}\right\|_{\mathrm{F}}^{2}=\left\|\mathbf{G}_{2}\right\|_{\mathrm{F}}^{2}=$ $m / 2$.

To get $\boldsymbol{\Phi}$ from (21), we need to calculate $\mathcal{J}_{i}^{\mathrm{H}} \mathbf{P}_{\mathrm{G}} \mathcal{J}_{i}$. With (17) and (27) in mind, one can find that, for $i \leq m, \mathcal{J}_{i}^{\mathrm{H}} \mathbf{P}_{\mathrm{G}} \mathcal{J}_{i}$ has the following structure:

$$
\mathcal{J}_{i}^{\mathrm{H}} \mathbf{P}_{\mathrm{G}} \mathcal{J}_{i}=\left[\begin{array}{cc}
\mathbf{A} & \mathbf{B} \\
\mathbf{B}^{*} & \mathbf{A}^{*}
\end{array}\right]
$$

where $\mathbf{A}, \mathbf{B}$ are the corresponding subblock matrices in $\mathcal{J}_{i}^{\mathrm{H}} \mathbf{P}_{\mathrm{G}} \mathcal{J}_{i}$. Therefore, only the first $M$ rows need to be calculated and stored in memory. Similarly, we know that, for $i>M$, the last $M$ rows of $\mathcal{J}_{i}^{\mathrm{H}} \mathbf{P}_{\mathrm{G}} \mathcal{J}_{i}$ can also be directly obtained from the conjugate of the first $M$ rows of $\mathcal{J}_{i-M+m}^{\mathrm{H}} \mathbf{P}_{\mathrm{G}} \mathcal{J}_{i-M+m}$. Therefore, these special properties can be utilized to reduce the storage burden and computational complexity of the proposed algorithm.

\section{E. Complexity Discussion}

It is well known that the dominant complexity component of subspace-based channel estimation lies in the singularvalue decomposition, which can be approximated by $O\left(N^{3}\right)$, where $N$ is the number of rows in the covariance matrix. Since our proposed method stacks the signal vector and its complex conjugate, the dimension of the covariance matrix is $2 M$, and the singular value decomposition (SVD) complexity is approximately $O\left((2 M)^{3}\right)$, which is twice as that for linear redundant-precoding algorithm [12] or the nonredundant precoding [14]. Therefore, the complexity of the proposed method is around eight times higher than these two algorithms. Nevertheless, since the block size of the CP-SC or OFDM is practically very large, e.g., $M=64$ in IEEE 802.11a [10], this factor eight does not appear to be a critical difference. Moreover, the complexity of the proposed method is much lower than that of virtual carrier-based method [13] since the latter must combine many consecutive blocks, which may form a covariance matrix with even higher dimension, as will be seen in the later simulations.

\section{Performance Analysis}

The deterministic CRB of the proposed estimator for real symbols with number $1 \leq m \leq M-1$ is derived next. The case when $m=M$ can similarly be obtained and will be omitted for brevity.
Clearly, the received snapshots satisfy the following deterministic model:

$$
\mathbf{x}(k) \sim \mathcal{N}\left\{\mathbf{H s}(k), \sigma^{2} \mathbf{I}\right\} .
$$

By suitably interchanging entries, the system model (4) can be rewritten as

$$
\mathbf{x}(k)=\mathbf{S}(k) \mathbf{h}+\mathbf{n}(k) .
$$

where $\mathbf{S}(k)$ is an $M \times(L+1)$ Toepoliz matrix with the first column $\mathbf{s}(k)$ and the first row $[\mathbf{s}(k)(1), \mathbf{s}(k)(M), \mathbf{s}(k)(M-1)$, $\ldots, \mathbf{s}(k)(M-L+2), \mathbf{s}(k)(M-L+1)]$. Here, $\mathbf{s}(k)(i)$ represents the $i$ th entry of $\mathbf{s}(k)$. Define

$$
\begin{aligned}
\boldsymbol{\Pi} & =\mathbf{H}(:, 1: m) \\
\mathbf{A} & =\mathbf{H}(:, m+1: M) \\
\mathbf{P}_{\mathbf{A}}^{\perp} & =\mathbf{I}-\mathbf{A}\left(\mathbf{A}^{\mathrm{H}} \mathbf{A}\right)^{-1} \mathbf{A}^{\mathrm{H}} \\
\mathbf{\Upsilon} & =\operatorname{Re}\left\{\boldsymbol{\Pi}^{\mathrm{H}} \mathbf{P}_{\mathbf{A}}^{\perp} \boldsymbol{\Pi}\right\} \\
\boldsymbol{\Delta}(k) & =\mathbf{S}(k)^{\mathrm{H}} \mathbf{P}_{\mathbf{A}}^{\perp} \mathbf{S}(k) \\
\mathbf{\Xi}(k) & =\mathbf{S}(k)^{\mathrm{H}} \mathbf{P}_{\mathbf{A}}^{\perp} \boldsymbol{\Pi} \\
\hat{\boldsymbol{\Delta}}(k) & =\left[\begin{array}{ll}
\operatorname{Re}\{\boldsymbol{\Delta}(k)\} & -\operatorname{Im}\{\boldsymbol{\Delta}(k)\} \\
\operatorname{Im}\{\boldsymbol{\Delta}(k)\} & \operatorname{Re}\{\boldsymbol{\Delta}(k)\}
\end{array}\right] .
\end{aligned}
$$

Suppose $N_{k}$ data blocks are received. The deterministic CRB of $\hat{\mathbf{h}} \triangleq\left[\operatorname{Re}\{\mathbf{h}\}^{\mathrm{T}}, \operatorname{Im}\{\mathbf{h}\}^{\mathrm{T}}\right]^{\mathrm{T}}$ is given as follows:

$$
\begin{aligned}
\mathrm{CRB}_{\hat{\mathbf{h}}}=\frac{\sigma^{2}}{2}\left(\sum _ { k = 1 } ^ { N _ { k } } \left(\hat{\boldsymbol{\Delta}}(k)-\left[\begin{array}{l}
\operatorname{Re}\{\boldsymbol{\Xi}(k)\} \\
\operatorname{Im}\{\boldsymbol{\Xi}(k)\}
\end{array}\right] \mathbf{\Upsilon}^{-1}\right.\right. \\
\left.\left.\times\left[\operatorname{Re}\{\boldsymbol{\Xi}(k)\}^{\mathrm{T}} \quad \operatorname{Im}\{\boldsymbol{\Xi}(k)\}^{\mathrm{T}}\right]\right)\right)^{\dagger} .
\end{aligned}
$$

Prooffor (32): See Appendix C.

\section{Simulation Results}

In this section, we examine the performance of the proposed estimator under various scenarios. The exponential power-delay profile

$$
E\left\{\left|h_{l}\right|^{2}\right\}=\exp (-l / 10), \quad l=0, \ldots, L
$$

is used for each channel tap. The phase of each channel ray is uniformly distributed over $[0,2 \pi)$. For all numerical examples, the parameters are taken as $M=16$ and $L=4$ and 200 data blocks are taken to obtain the channel matrix unless otherwise mentioned. Note that 200 data blocks are commonly used in subspace-based channel estimation [11]-[13]. Moreover, all the results are averaged over $N_{d}=100$ Monte Carlo runs. The root-normalized estimation mean-square error (RNMSE) is defined as

$$
\text { RNMSE }=\sqrt{\frac{1}{N_{d}} \sum_{d=1}^{N_{d}} \frac{\left\|\ddot{\mathbf{h}}_{d}-\mathbf{h}\right\|^{2}}{\|\mathbf{h}\|^{2}}}
$$

where the subscript $d$ refers to the $d$ th simulation run. 
TABLE I

NUMERICAL STUdy ON condi FOR DifFERENT VALUES OF $m$

\begin{tabular}{|c|c|c|c|c|c|c|c|c|}
\hline condi & $1 \sim 10$ & $10 \sim 10^{2}$ & $10^{2} \sim 10^{3}$ & $10^{3} \sim 10^{4}$ & $10^{4} \sim 10^{5}$ & $10^{5} \sim 10^{6}$ & $10^{6} \sim 10^{7}$ & $10^{7} \sim+\infty$ \\
\hline $\mathrm{m}=1$ & 419380 & 520698 & 54225 & 5141 & 491 & 60 & 5 & 0 \\
\hline $\mathrm{m}=2$ & 390075 & 565827 & 40224 & 3474 & 361 & 36 & 3 & 0 \\
\hline $\mathrm{m}=4$ & 293086 & 699875 & 7011 & 28 & 0 & 0 & 0 & 0 \\
\hline $\mathrm{m}=8$ & 161163 & 836397 & 2440 & 0 & 0 & 0 & 0 & 0 \\
\hline $\mathrm{m}=12$ & 116876 & 879083 & 4041 & 0 & 0 & 0 & 0 & 0 \\
\hline $\mathrm{m}=14$ & 100178 & 895734 & 4088 & 0 & 0 & 0 & 0 & 0 \\
\hline $\mathrm{m}=15$ & 84132 & 910636 & 5232 & 0 & 0 & 0 & 0 & 0 \\
\hline $\mathrm{m}=16$ & 47467 & 441482 & 322347 & 126618 & 42126 & 13567 & 4345 & 2048 \\
\hline
\end{tabular}

\section{A. Example 1: Channel Ambiguity}

In this example, we numerically study the channel-estimation ambiguity of the proposed algorithm as a function of the number of the real symbols $m$. The process is described as follows. We randomly generate $10^{6}$ different channel vectors $\mathbf{h}$ and construct the corresponding channel matrices $\breve{\mathbf{H}}$ by using different values of $m$. Applying SVD on these channel matrices, we obtain the exact noise subspace that is orthogonal to $\breve{\mathbf{H}}$ and the matrix $\boldsymbol{\Phi}$, following (21). The smallest singular value of $\boldsymbol{\Phi}$ is zero; if the second smallest singular value of $\boldsymbol{\Phi}$ is also zero, then ambiguity occurs. However, due to computer roundingoff errors, the second smallest singular value of $\boldsymbol{\Phi}$ would not be zero even when ambiguity happens. We now choose a new criterion in judging the channel ambiguity. Define

$$
\overline{\text { condi }}=\frac{\text { largest singular value of } \boldsymbol{\Phi}}{\text { second smallest singular value of } \boldsymbol{\Phi}} .
$$

The numerical study on condi for different values of $m$ is provided in Table I. We see that condi is quite big for some cases in $m=1, m=2$, and, particularly, $m=16$. However, we cannot say whether the ambiguity will definitely occur even for these large condi because, as long as the second smallest singular value is nonzero, ${ }^{2}$ the channel ambiguity should not occur under noise-free case. What we can conclude by observing Table I are the following.

1) The value of $m$ that can give the smallest chance for channel ambiguity belongs to $\{8, \ldots, 15\}$.

2) The chances for channel ambiguity increases when $m$ decreases from eight. Nevertheless, the probability of the channel ambiguity, if any, is quite small.

3) The full real transmission is more vulnerable to noise and has the highest chance of channel-estimation ambiguity if noise is involved, particularly at the lower signal-to-noise ratio (SNR) region.

\section{B. Example 2: Comparing With the Existing Methods}

In this example, we compare the proposed method with the redundant-precoding method [12], the virtual-carrier-based method [13], the nonredundant-precoding method [14], and the training-based method. For a fair comparison, symbols on different time slots are independently generated, and their power is adjusted such that the covariance of $\mathbf{s}(k)$ is a multiple of an identity matrix. For the proposed method and the training-based

\footnotetext{
${ }^{2}$ May be due to computer rounding-off errors.
}

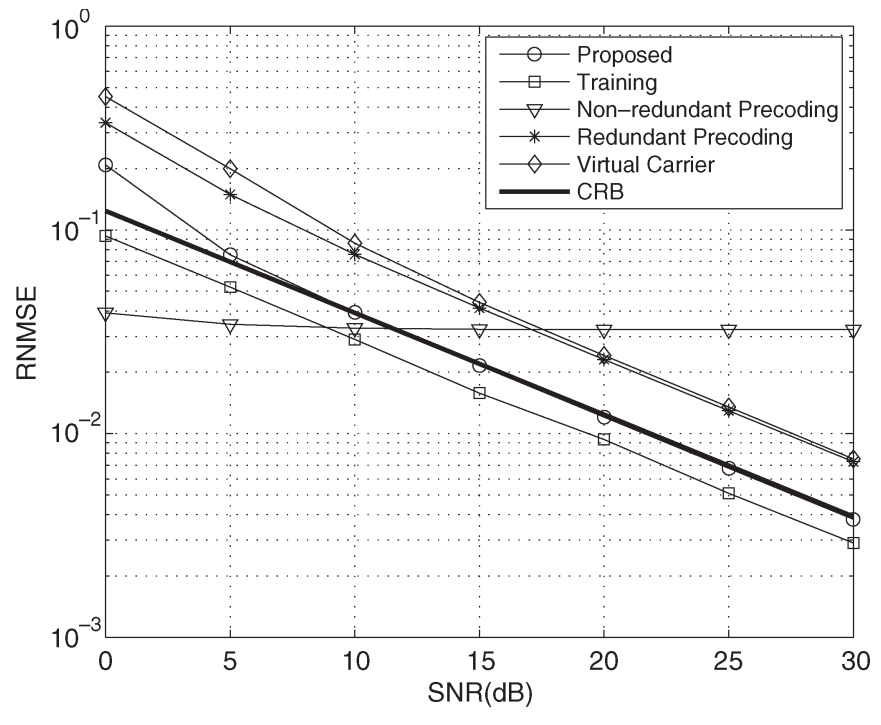

Fig. 2. RNMSE of the proposed method and of existing methods as a function of SNR.

method, the data block is transmitted in the time domain; the first symbol in each block is 4-PAM, and others are 16-QAM. For the methods in [12]-[14], the data block is transmitted in the frequency domain. As suggested in [12], an $M \times(M-1)$ precoding matrix, with entries randomly taken from $\{+1,-1\}$, is used for the redundant-precoding method. Therefore, in each OFDM block, one data symbol is lost to provide redundancy. For the method in [13], we also adopt the smallest redundancy and transmit only on $M-1$ subcarriers. From [13], we know that five consecutive OFDM blocks need to be combined to form the covariance matrix that has a dimension of $5 M \times 5 M$. Since, in each transmission block, the proposed method adds a half symbol of redundancy, we take 100 symbols for the training-based method. In Fig. 2, the RNMSEs of channel estimation versus the SNR of these four different algorithms are displayed. The deterministic CRB for the proposed method is also displayed.

Fig. 2 shows that the proposed method outperforms the redundant-precoding method and the virtual-carrier-based method, although the latter two methods have twice the redundancy as that in the proposed method. Moreover, the proposed method performs better than the nonredundant-precoding method at relatively high SNR. The training-based method performs better than the proposed method. However, for cases where a training sequence is not applicable, e.g., noncooperative transmission, blind methods would be the only choice. 


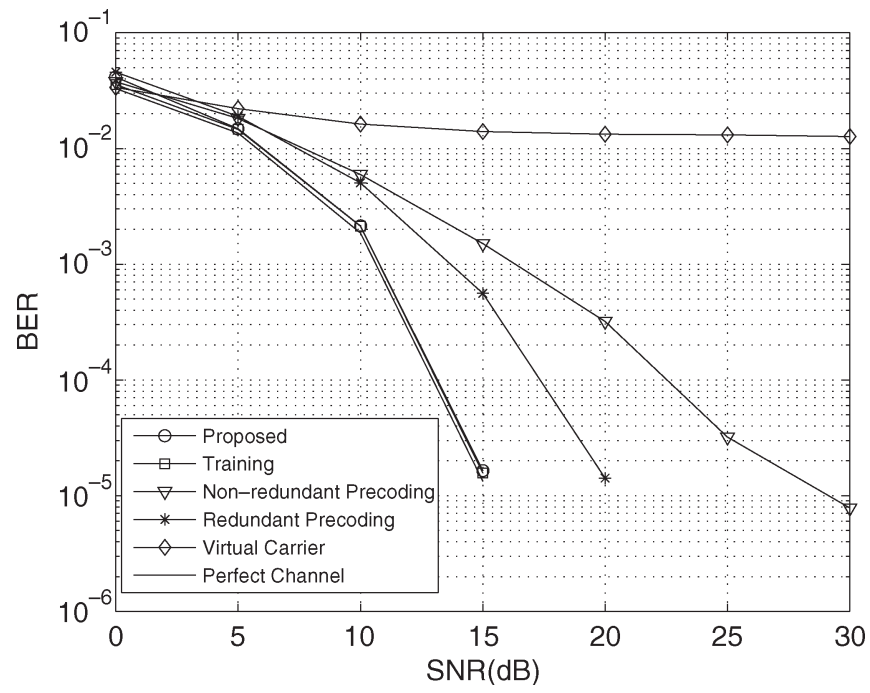

Fig. 3. BER of the proposed method and of existing methods as a function of SNR.

Moreover, the performance difference between the proposed method and the training-based method is quite small. The performance of the proposed algorithm matches well with the $\mathrm{CRB}$, a phenomenon that is quite important because it says that, for the considered real combined system, our blind algorithm gives the best performance as compared to all other blindchannel-estimation methods, if any.

MMSE detection is applied at the receiver, and the bit-errorrate (BER) performance of these five methods is shown in Fig. 3. The proposed method not only performs comparably to the training-based one but also significantly outperforms the other two precoding methods. The performance curve for perfect channel knowledge is also plotted for a comparison. Both the proposed method and the training-based method match with the perfect curve well. From the simulations, we find that the channel matrix of the virtual-carrier-based method is ill conditioned. ${ }^{3}$ This is the reason why the virtual-carrier-based method gives the worst BER performance.

\section{Example 3: Different Numbers of Real Symbols}

In this example, we examine the performance of the proposed method for $m=1,2,4,8$, and 16 , respectively. It is expected that, as the number of real symbols increases, channel-estimation accuracy should increase. Fig. 4 shows the RNMSEs of channel estimation versus the SNR of these five different cases, along with the deterministic CRBs. The corresponding BER performances are shown in Fig. 5. From Fig. 4, we see that, as the number of the real symbols increases from one to eight, the RNMSE performance becomes better. It should be mentioned that the performance for $m \in$ $\{9,10, \ldots, 15\}$ is similar to that for $m=8$. However, these performance lines are not shown. It is also noted that the algorithm under $m=16$, surprisingly, gives the worst performance. In fact, the phenomenon shown in Fig. 4 is quite consistent with that in Table I, which implicitly indicates the

\footnotetext{
${ }^{3}$ Condition number is around $10^{6}$.
}

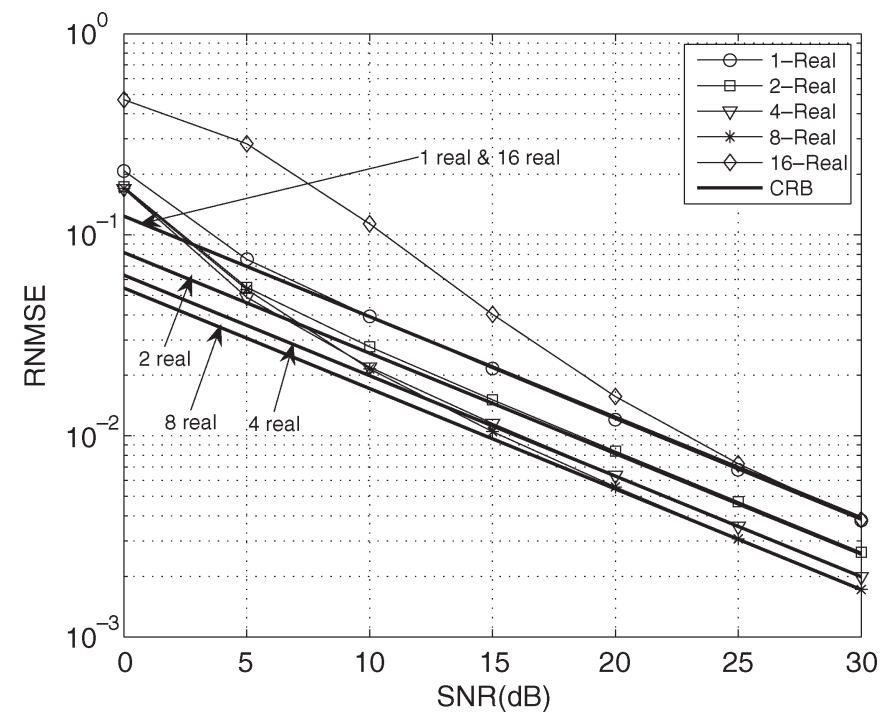

Fig. 4. RNMSE versus SNR for different numbers of real symbols.

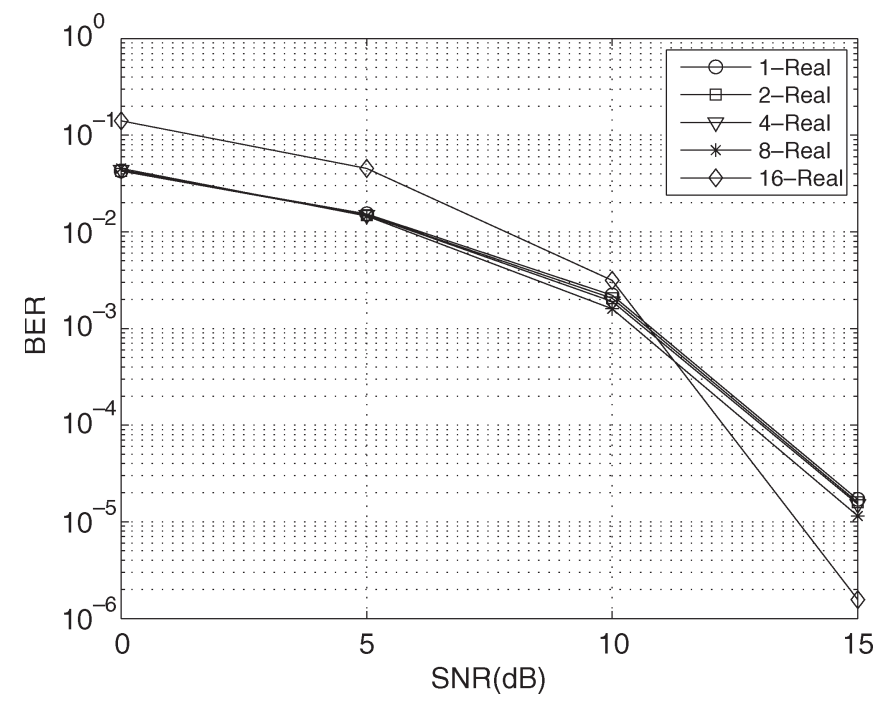

Fig. 5. BER versus SNR for different numbers of real symbols.

bad performance for $m=16$, the similar performance for $m \in\{8, \ldots, 15\}$, and the gradually decreasing performance for $m$ from eight to one. From Fig. 5, larger $m$ yields better performance. This is because of the assumption of the same symbol energy for both real and complex constellations. Namely, the Euclidean distance between points in 4-PAM constellation is greater than that in 16-QAM constellation. However, $m=16$ appears as a special case, where the BER performance only becomes better at high SNR. This is a direct result from the fact that channel estimation becomes better only at high SNR, as shown in Fig. 4. Moreover, we see that one real symbol is already enough to yield reliable channel estimation and, consequently, reliable symbol detection. Therefore, if the original channel matrix is square, then we suggest to deliberately introduce only one real symbol. A side conclusion from these two numerical results is that full real transmissions in SC-CP systems are not recommended at low SNR for the proposed method. 


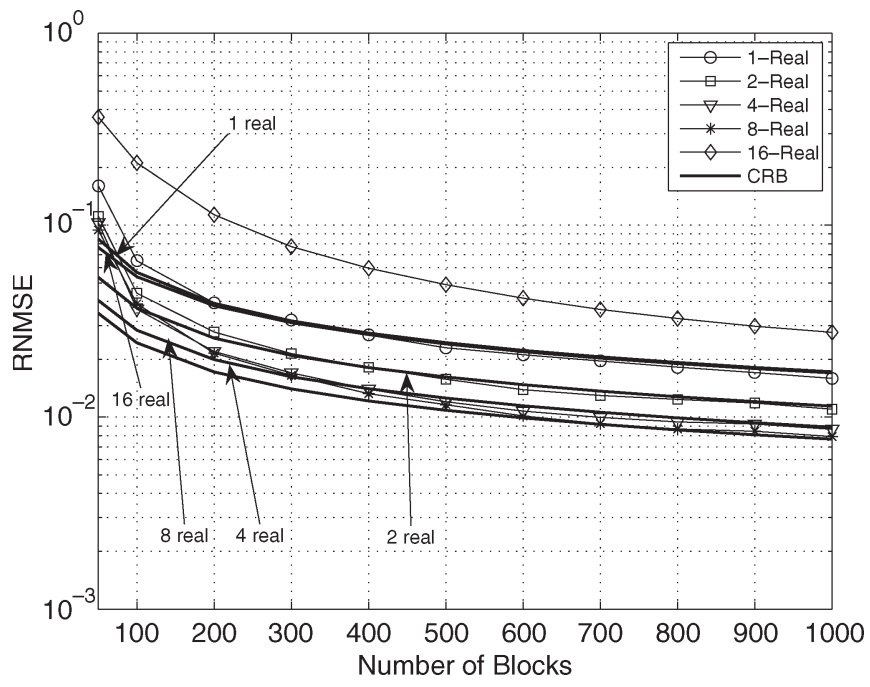

Fig. 6. RNMSE versus number of data blocks for different numbers of real symbols.

We examine next how the proposed estimator performs under different numbers of received-signal blocks. The SNR, in this example, is fixed at $10 \mathrm{~dB}$. It is expected that, as the number of the signal blocks increases, the estimate of the covariance matrix $\mathbf{R}^{\prime}$ becomes accurate, which results in better estimation of $\mathbf{h}$. Fig. 6 shows the RNMSEs of channel estimation versus the number of blocks of four curves for $m=1,2,4,8$, and 16 . The CRBs are displayed as well. The estimates with more than 200 blocks give satisfactory performance.

\section{Example 4: Real Symbol at Different Position}

It is also interesting to examine the impact of the position of real symbols on the performance of the proposed estimator. In this example, one real symbol is assumed at the positions $1,2,4,8$, and 16 , respectively. The RNMSEs versus the SNR performance of the proposed estimator is shown in Fig. 7 for the five different cases; the five curves almost overlap with each other, and the difference is quite small. In conclusion, while the location of the real symbols does affect the performance, the impact is small.

\section{E. Example 5: Comparison With Traditional Blind Method}

In this example, we apply our proposed algorithm to the zeropadding SISO SC transmission and examine the performance comparison between the direct channel-estimation method [19] and our proposed method. Similar block size and channel vectors are used as in the previous examples. Therefore, four zeros are added at the end of each block. In this case, the channel matrix is already tall, and no real symbols are needed to construct a tall matrix. However, our interest lies in the case where there already exist real symbols for certain demands and how the real symbols will affect the channel-estimation results. We assume that $m=2,4,8$, and 16 real symbols exist in each data block, which are obtained from the 4-PAM constellation and the other symbols are taken from 16-QAM. The traditional subspace-based method in [19] directly operates on the covariance matrix of the received data block, while the proposed

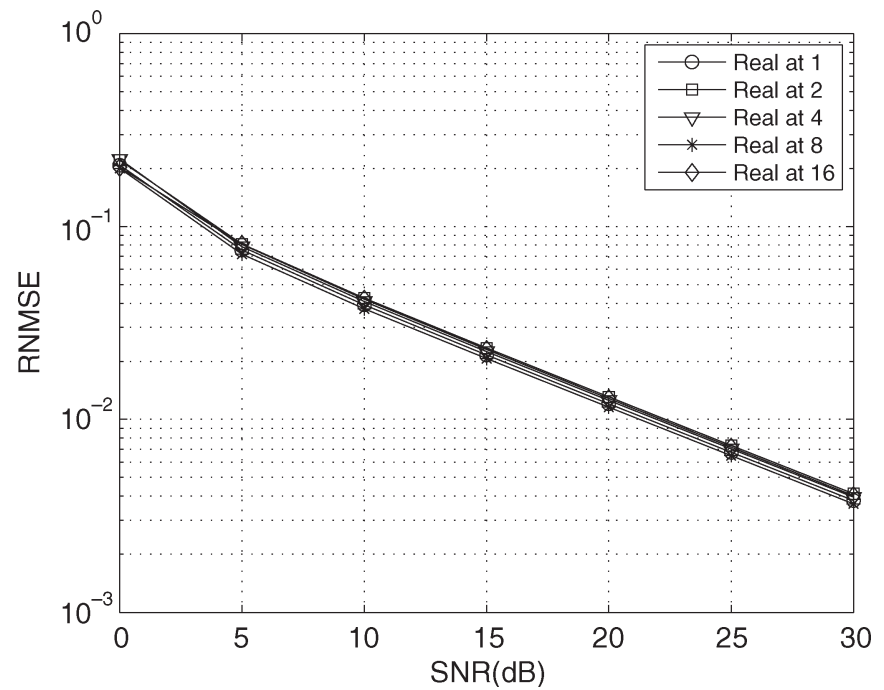

Fig. 7. RNMSE versus SNR for different positions of real symbols.

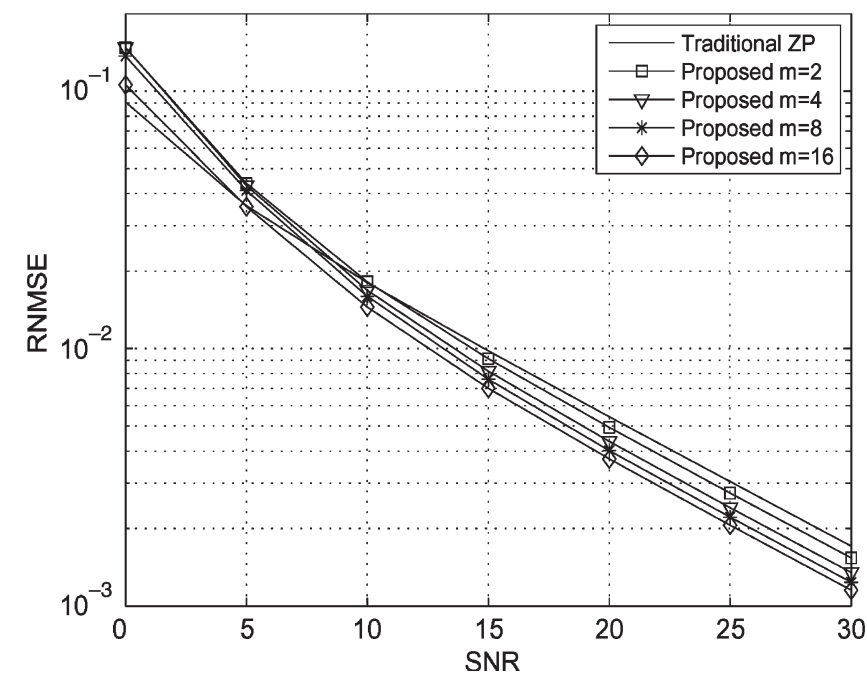

Fig. 8. Comparison of the proposed method with traditional subspace-based method.

algorithm considers the effect of both the received signals and its conjugate part. The RNMSEs versus the SNR performance is shown in Fig. 8. It can be seen that our proposed method is better than the traditional subspace method at relatively higher SNR region. In particular, when $m=16$, there is nearly 4-dB SNR gain by using the proposed method. This is because we fully exploit the inherent properties in the transmitted data. Note that the case $m=16$ does not give the worst performance here since the channel-matrix structure is totally different from that in CP-SC system. This more or less supports our previous conjecture that the bad performance for $m=16$ in CP-SC may come from the bad condition of the channel matrix (see Table I). We reemphasize that, when real symbols exist, we should avoid direct estimation but combine the signal characteristic into the channel-estimation algorithm.

\section{F. Example 6: Performance With Large Block Size}

In this example, we evaluate the proposed algorithm under more practical parameters taken from IEEE 802.11a [10], 


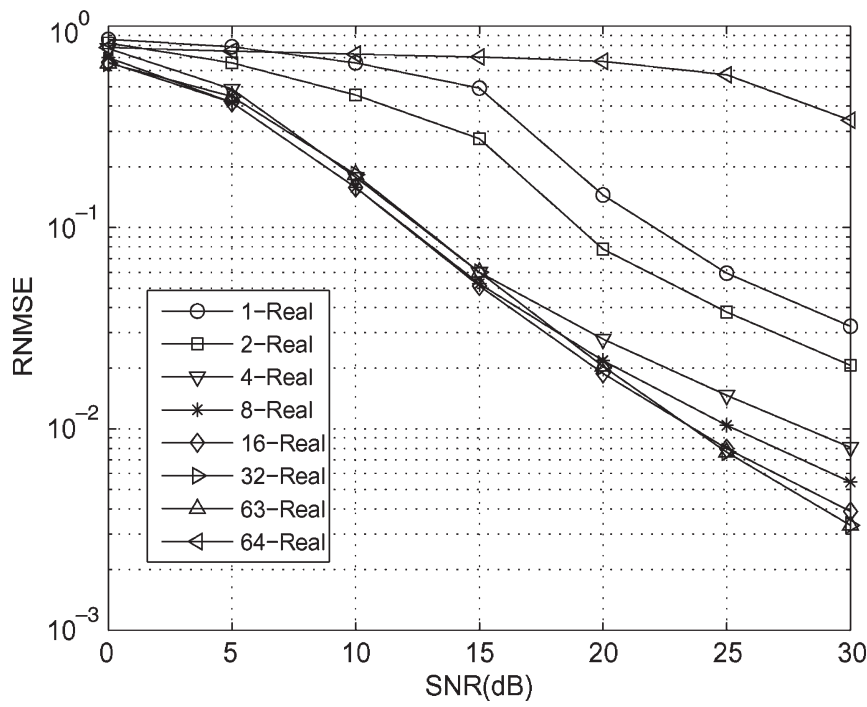

Fig. 9. RNMSE versus SNR for different numbers of real symbols-large block size.

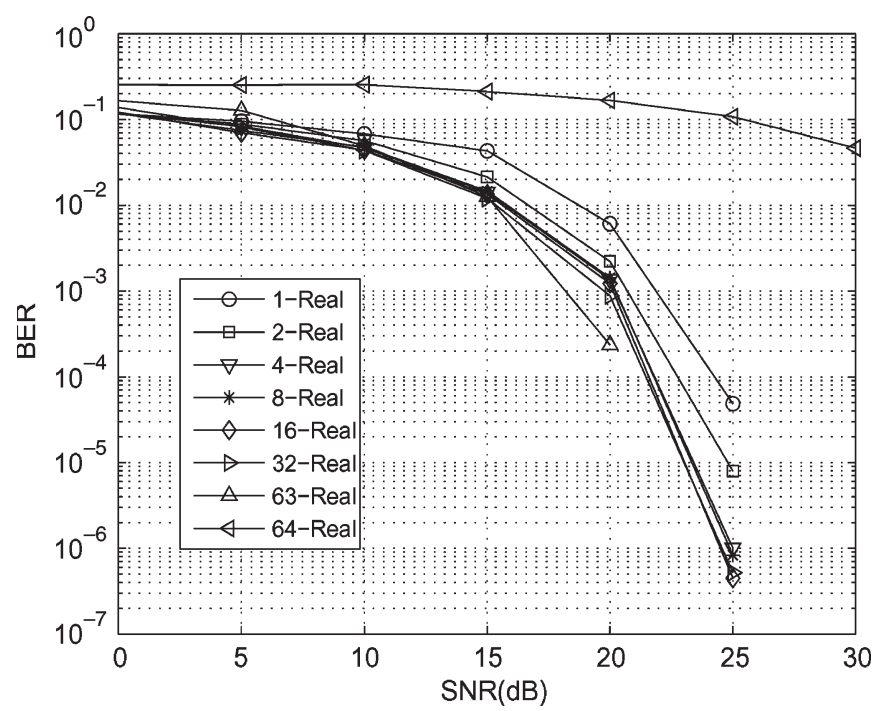

Fig. 10. BER versus SNR for different numbers of real symbols-large block size.

where $M$ and $L$ are selected as 64 and 16 , respectively. We also consider the worst case that the channel taps are as long as $L+1=17$ while still following the model (33). The channelestimation RNMSEs and the BER performance for $m=1,2,4$, 8, 16, 32, 63, and 64 are shown, respectively, in Figs. 9 and 10. It is shown that the proposed algorithm is also effective for transmissions with larger block size. This is to be expected since there is no specific size limitation in the proposed algorithm. Similar behaviors are observed from Figs. 4 and 5 in that both the RNMSE and BER decrease with the increase of the number of the real symbols, except for the special full real case. This example validates the proposed algorithm for parameters under practical transmissions.

\section{ConClusion}

In this paper, the characteristics of real symbols are fully exploited in the subspace-based blind channel estimation for
CP-SC transmissions. The proposed method not only requires less redundancy as compared to the previous techniques in [12] and [13] but also increases the channel-estimation accuracy as compared to the traditional subspace-based method since the latter does not fully exploit the signal-structure information. The proposed method also reduces the phase ambiguity in the channel estimation to sign ambiguity only, which facilitates the detection of symbols. Several mathematical studies, including the channel-ambiguity discussions, ways to reduce the storage burden, and the deterministic CRB, are provided to support this paper. Simulations clearly demonstrate the effectiveness of the proposed real-symbol combined method, as well as its many advantages over the exiting methods.

\section{APPENDIX A \\ PROOF OF THEOREM 3}

If all the transmitted symbols are real, matrix $\breve{\mathbf{H}}$ can be written as

$$
\breve{\mathbf{H}}=\left[\mathbf{H}^{\mathrm{T}}, \mathbf{H}^{\mathrm{H}}\right]^{\mathrm{T}} .
$$

Let $\rho$ be an arbitrary vector and let $\mathcal{A}$ be constructed from $\rho$ the same way as $\breve{\mathbf{H}}$ is constructed from $\mathbf{h}$. Suppose matrices $\mathcal{A}$ and $\breve{\mathbf{H}}$ span the same space, namely

$$
\mathcal{A}=\breve{\mathbf{H B}}
$$

where $\mathbf{B}$ is an unknown matrix. Then, we only need to prove that $\boldsymbol{\rho}=\gamma \mathbf{h}$ for some scalar $\gamma$.

The first column of $\mathcal{A}$ satisfies

$$
\mathcal{A}(:, 1)=\breve{\mathbf{H B}}(:, 1)=\breve{\mathbf{H} b}
$$

where $\mathbf{b}$ is the first column of matrix $\mathbf{B}$. By properly rearranging the elements in (38), it follows that

$$
\left[\begin{array}{c}
\boldsymbol{\rho} \\
\boldsymbol{\rho}^{*} \\
\mathbf{0}_{(M-L-1) \times 1} \\
\mathbf{0}_{(M-L-1) \times 1}
\end{array}\right]=\left[\begin{array}{cc}
\mathbf{h} & \mathbf{D}_{1} \\
\mathbf{h}^{*} & \mathbf{D}_{2} \\
\mathbf{0}_{(M-L-1) \times 1} & \mathcal{H} \\
\mathbf{0}_{(M-L-1) \times 1} & \mathcal{H}^{*}
\end{array}\right]\left[\begin{array}{c}
\mathbf{b}(1) \\
\mathbf{b}(2: M)
\end{array}\right]
$$

where the $(M-1-L) \times(M-1)$ matrix $\mathcal{H}$ has the form

$$
\mathcal{H}=\left[\begin{array}{ccccc}
h_{L} & \cdots & h_{0} & \cdots & 0 \\
\vdots & \ddots & \ddots & \ddots & \vdots \\
0 & \cdots & h_{L} & \cdots & h_{0}
\end{array}\right]
$$

It is readily known from [11] that if $\sum_{l=0}^{L} h_{l} z^{-l}$ and $\sum_{l=0}^{L} h_{l}^{*} z^{-l}$ has no common zero and $2(M-1-L) \geq M-$ 1 , then the matrix

$$
\tilde{\mathcal{H}} \triangleq\left[\begin{array}{c}
\mathcal{H} \\
\mathcal{H}^{*}
\end{array}\right]
$$

is a full column rank matrix. The first condition equivalently says that no root of $h(z)=\sum_{l=0}^{L} h_{l} z^{-l}$ is real valued and no two roots are conjugate of each other. If these conditions 
are satisfied, $\tilde{\mathcal{H}}$ is assured to be a full column rank matrix Then, from

$$
\left[\begin{array}{l}
\mathbf{0}_{(M-L-1) \times 1} \\
\mathbf{0}_{(M-L-1) \times 1}
\end{array}\right]=\left[\begin{array}{c}
\mathcal{H} \\
\mathcal{H}^{*}
\end{array}\right] \mathbf{b}(2: M)
$$

we know that $\mathbf{b}(2: M)$ is a zero vector. Consequently

$$
\left[\begin{array}{c}
\boldsymbol{\rho} \\
\boldsymbol{\rho}^{*}
\end{array}\right]=\left[\begin{array}{c}
\mathbf{h} \\
\mathbf{h}^{*}
\end{array}\right] \mathbf{b}(1)
$$

and $\boldsymbol{\rho}=\gamma \mathbf{h}$ with $\gamma=\mathbf{b}(1)$ is a real scalar.

\section{APPENDIX B}

\section{PROOF OF THEOREM 4}

From the previous discussion, we know that

$$
\left[\mathbf{G}_{1}^{\mathrm{H}}, \mathbf{G}_{2}^{\mathrm{H}}\right] \breve{\mathbf{H}}=\mathbf{0} .
$$

Let

$$
\breve{\mathbf{H}}^{\prime}=\left[\breve{\mathbf{H}}(M+1: 2 M,:)^{\mathrm{T}}, \breve{\mathbf{H}}(1: M,:)^{\mathrm{T}}\right]^{\mathrm{T}}
$$

represent the interchange of the first and the last $M$ rows of $\breve{\mathbf{H}}$. From (44), we know that

$$
\left[\mathbf{G}_{2}^{\mathrm{H}}, \mathbf{G}_{1}^{\mathrm{H}}\right] \breve{\mathbf{H}}^{\prime}=\mathbf{0}
$$

By taking the complex conjugate of both side of (46), we obtain

$$
\left[\mathbf{G}_{2}^{\mathrm{T}}, \mathbf{G}_{1}^{\mathrm{T}}\right] \breve{\mathbf{H}}=\tilde{\mathbf{G}} \breve{\mathbf{H}}=\mathbf{0} .
$$

Obviously, $\tilde{\mathbf{G}}$ is a $2 M \times m$ orthonormal matrix. Since matrix $\breve{\mathbf{H}}$ is of full column rank, matrix $\tilde{\mathbf{G}}$ will also span the noise subspace. From the uniqueness of the projection matrix onto a subspace, one can readily conclude that

$$
\mathbf{P}_{\mathbf{G}}=\mathbf{G G}^{\mathrm{H}}=\tilde{\mathbf{G}} \tilde{\mathbf{G}}^{\mathrm{H}} .
$$

\section{APPENDIX C}

\section{Proof OF DETERMINISTIC CRB}

We omit the index $k$ in this part for a clear demonstration. It is well known that, for the single-vector observation

$$
\mathbf{x}=\mathbf{H s}+\mathbf{n}=\mathbf{S h}+\mathbf{n}
$$

the Fisher's Information Matrix (FIM) for the signal parameter vector

$$
\begin{aligned}
\boldsymbol{\theta}=\left[\operatorname{Re}\{\mathbf{h}\}^{\mathrm{T}}, \operatorname{Im}\{\mathbf{h}\}^{\mathrm{T}}, \mathbf{s}(1: m)^{\mathrm{T}}\right. & \\
& \left.\operatorname{Re}\{\mathbf{s}(m+1: M)\}^{\mathrm{T}}, \operatorname{Im}\{\mathbf{s}(m+1: M)\}^{\mathrm{T}}\right]^{\mathrm{T}}
\end{aligned}
$$

is given by [20]

$$
\mathbf{J}=\frac{2}{\sigma^{2}} \operatorname{Re}\left\{\boldsymbol{\Sigma}^{\mathrm{H}} \boldsymbol{\Sigma}\right\}
$$

where

$$
\begin{aligned}
\boldsymbol{\Sigma} \triangleq & {\left[\frac{\partial \mathbf{H s}}{\partial \boldsymbol{\theta}^{\mathrm{T}}}\right] } \\
= & {\left[\frac{\partial \mathbf{H s}}{\partial \operatorname{Re}\{\mathbf{h}\}^{\mathrm{T}}}, \frac{\partial \mathbf{H s}}{\partial \operatorname{Im}\{\mathbf{h}\}^{\mathrm{T}}}, \frac{\partial \mathbf{H s}}{\partial \mathbf{s}(1: m)^{\mathrm{T}}},\right.} \\
& \left.\frac{\partial \mathbf{H s}}{\partial \operatorname{Re}\{\mathbf{s}(m+1: M)\}^{\mathrm{T}}}, \frac{\partial \mathbf{H s}}{\partial \operatorname{Im}\{\mathbf{s}(m+1: M)\}^{\mathrm{T}}}\right] .
\end{aligned}
$$

It can be obtained straightforwardly that

$$
\begin{aligned}
\frac{\partial \mathbf{H} \mathbf{s}}{\partial \operatorname{Re}\{\mathbf{h}\}^{\mathrm{T}}} & =\mathbf{S} \\
\frac{\partial \mathbf{H} \mathbf{s}}{\partial \operatorname{Im}\{\mathbf{h}\}^{\mathrm{T}}} & =j \mathbf{S} \\
\frac{\partial \mathbf{H} \mathbf{s}}{\partial \mathbf{s}(1: m)^{\mathrm{T}}} & =\mathbf{\Pi} \\
\frac{\partial \mathbf{H} \mathbf{s}}{\partial \operatorname{Re}\{\mathbf{s}(m+1: M)\}^{\mathrm{T}}} & =\mathbf{A} \\
\frac{\partial \mathbf{H} \mathbf{s}}{\partial \operatorname{Im}\{\mathbf{s}(m+1: M)\}^{\mathrm{T}}} & =j \mathbf{A} .
\end{aligned}
$$

Then, the structure of the FIM is given in (54), shown at the bottom of the page.

From [20], we know that, for blind channel estimation, the FIM is singular, such that, its inverse does not exist. Then, some constraints should be utilized to make $\mathbf{J}$ a nonsingular matrix. Instead of taking any specific constraint, we use the minimal constrained CRB, which is defined as in [20].

Lemma 1 [20]: Suppose that the FIM for $\boldsymbol{\theta}=\left[\boldsymbol{\theta}_{1}^{\mathrm{T}}, \boldsymbol{\theta}_{2}^{\mathrm{T}}\right]^{\mathrm{T}}$ is

$$
\mathbf{J}=\frac{2}{\sigma^{2}}\left[\begin{array}{ll}
\mathbf{J}_{\boldsymbol{\theta}_{1} \boldsymbol{\theta}_{1}} & \mathbf{J}_{\boldsymbol{\theta}_{1} \boldsymbol{\theta}_{2}} \\
\mathbf{J}_{\boldsymbol{\theta}_{2} \boldsymbol{\theta}_{1}} & \mathbf{J}_{\boldsymbol{\theta}_{2} \boldsymbol{\theta}_{2}}
\end{array}\right]
$$

$$
\mathbf{J}=\frac{2}{\sigma^{2}} \operatorname{Re}\left\{\boldsymbol{\Sigma}^{\mathrm{H}} \boldsymbol{\Sigma}\right\}=\frac{2}{\sigma^{2}}\left[\begin{array}{rrrrr}
\operatorname{Re}\left\{\mathbf{S}^{\mathrm{H}} \mathbf{S}\right\} & -\operatorname{Im}\left\{\mathbf{S}^{\mathrm{H}} \mathbf{S}\right\} & \operatorname{Re}\left\{\mathbf{S}^{\mathrm{H}} \boldsymbol{\Pi}\right\} & \operatorname{Re}\left\{\mathbf{S}^{H} \mathbf{A}\right\} & -\operatorname{Im}\left\{\mathbf{S}^{\mathrm{H}} \mathbf{A}\right\} \\
\operatorname{Im}\left\{\mathbf{S}^{\mathrm{H}} \mathbf{S}\right\} & \operatorname{Re}\left\{\mathbf{S}^{\mathrm{H}} \mathbf{S}\right\} & \operatorname{Im}\left\{\mathbf{S}^{\mathrm{H}} \boldsymbol{\Pi}\right\} & \operatorname{Im}\left\{\mathbf{S}^{\mathrm{H}} \mathbf{A}\right\} & \operatorname{Re}\left\{\mathbf{S}^{\mathrm{H}} \mathbf{A}\right\} \\
\operatorname{Re}\left\{\mathbf{\Pi}^{\mathrm{H}} \mathbf{S}\right\} & -\operatorname{Im}\left\{\mathbf{\Pi}^{\mathrm{H}} \mathbf{S}\right\} & \operatorname{Re}\left\{\boldsymbol{\Pi}^{\mathrm{H}} \boldsymbol{\Pi}\right\} & \operatorname{Re}\left\{\mathbf{\Pi}^{\mathrm{H}} \mathbf{A}\right\} & -\operatorname{Im}\left\{\mathbf{\Pi}^{\mathrm{H}} \mathbf{A}\right\} \\
\operatorname{Re}\left\{\mathbf{A}^{\mathrm{H}} \mathbf{S}\right\} & -\operatorname{Im}\left\{\mathbf{A}^{\mathrm{H}} \mathbf{S}\right\} & \operatorname{Re}\left\{\mathbf{A}^{\mathrm{H}} \boldsymbol{\Pi}\right\} & \operatorname{Re}\left\{\mathbf{A}^{\mathrm{H}} \mathbf{A}\right\} & -\operatorname{Im}\left\{\mathbf{A}^{\mathrm{H}} \mathbf{A}\right\} \\
\operatorname{Im}\left\{\mathbf{A}^{\mathrm{H}} \mathbf{S}\right\} & \operatorname{Re}\left\{\mathbf{A}^{\mathrm{H}} \mathbf{S}\right\} & \operatorname{Im}\left\{\mathbf{A}^{\mathrm{H}} \boldsymbol{\Pi}\right\} & \operatorname{Im}\left\{\mathbf{A}^{\mathrm{H}} \mathbf{A}\right\} & \operatorname{Re}\left\{\mathbf{A}^{\mathrm{H}} \mathbf{A}\right\}
\end{array}\right]
$$


and assume $\mathbf{J}$ is singular but $\mathbf{J}_{\boldsymbol{\theta}_{2} \boldsymbol{\theta}_{2}}$ is nonsingular. Then, the minimal constrained CRB for $\boldsymbol{\theta}_{1}$ separately is

$$
\mathrm{CRB}_{\boldsymbol{\theta}_{1}}=\frac{\sigma^{2}}{2}\left[\mathbf{J}_{\boldsymbol{\theta}_{1} \boldsymbol{\theta}_{1}}-\mathbf{J}_{\boldsymbol{\theta}_{1} \boldsymbol{\theta}_{2}} \mathbf{J}_{\boldsymbol{\theta}_{2} \boldsymbol{\theta}_{2}}^{-1} \mathbf{J}_{\boldsymbol{\theta}_{2} \boldsymbol{\theta}_{1}}\right]^{\dagger} \text {. }
$$

Letting $\boldsymbol{\theta}_{1}=\left[\operatorname{Re}\{\mathbf{h}\}^{\mathrm{T}}, \operatorname{Im}\{\mathbf{h}\}^{\mathrm{T}}\right]^{\mathrm{T}}=\hat{\mathbf{h}}$, it follows that

$$
\begin{aligned}
\mathbf{J}_{\boldsymbol{\theta}_{1} \boldsymbol{\theta}_{1}}= & {\left[\begin{array}{lrr}
\operatorname{Re}\left\{\mathbf{S}^{\mathrm{H}} \mathbf{S}\right\} & -\operatorname{Im}\left\{\mathbf{S}^{\mathrm{H}} \mathbf{S}\right\} \\
\operatorname{Im}\left\{\mathbf{S}^{\mathrm{H}} \mathbf{S}\right\} & \operatorname{Re}\left\{\mathbf{S}^{\mathrm{H}} \mathbf{S}\right\}
\end{array}\right] } \\
\mathbf{J}_{\boldsymbol{\theta}_{1} \boldsymbol{\theta}_{2}}= & {\left[\begin{array}{lrr}
\operatorname{Re}\left\{\mathbf{S}^{\mathrm{H}} \boldsymbol{\Pi}\right\} & \operatorname{Re}\left\{\mathbf{S}^{\mathrm{H}} \mathbf{A}\right\} & -\operatorname{Im}\left\{\mathbf{S}^{\mathrm{H}} \mathbf{A}\right\} \\
\operatorname{Im}\left\{\mathbf{S}^{\mathrm{H}} \boldsymbol{\Pi}\right\} & \operatorname{Im}\left\{\mathbf{S}^{\mathrm{H}} \mathbf{A}\right\} & \operatorname{Re}\left\{\mathbf{S}^{\mathrm{H}} \mathbf{A}\right\}
\end{array}\right] } \\
\mathbf{J}_{\boldsymbol{\theta}_{2} \boldsymbol{\theta}_{2}}= & {\left[\begin{array}{lrr}
\operatorname{Re}\left\{\boldsymbol{\Pi}^{\mathrm{H}} \boldsymbol{\Pi}\right\} & \operatorname{Re}\left\{\boldsymbol{\Pi}^{\mathrm{H}} \mathbf{A}\right\} & -\operatorname{Im}\left\{\boldsymbol{\Pi}^{\mathrm{H}} \mathbf{A}\right\} \\
\operatorname{Re}\left\{\mathbf{A}^{\mathrm{H}} \boldsymbol{\Pi}\right\} & \operatorname{Re}\left\{\mathbf{A}^{\mathrm{H}} \mathbf{A}\right\} & -\operatorname{Im}\left\{\mathbf{A}^{\mathrm{H}} \mathbf{A}\right\} \\
\operatorname{Im}\left\{\mathbf{A}^{\mathrm{H}} \mathbf{\Pi}\right\} & \operatorname{Im}\left\{\mathbf{A}^{\mathrm{H}} \mathbf{A}\right\} & \operatorname{Re}\left\{\mathbf{A}^{\mathrm{H}} \mathbf{A}\right\}
\end{array}\right] } \\
\mathbf{J}_{\boldsymbol{\theta}_{2} \boldsymbol{\theta}_{1}}= & {\left[\begin{array}{lr}
\operatorname{Re}\left\{\boldsymbol{\Pi}^{\mathrm{H}} \mathbf{S}\right\} & -\operatorname{Im}\left\{\mathbf{\Pi}^{\mathrm{H}} \mathbf{S}\right\} \\
\operatorname{Re}\left\{\mathbf{A}^{\mathrm{H}} \mathbf{S}\right\} & -\operatorname{Im}\left\{\mathbf{A}^{\mathrm{H}} \mathbf{S}\right\} \\
\operatorname{Im}\left\{\mathbf{A}^{\mathrm{H}} \mathbf{S}\right\} & \operatorname{Re}\left\{\mathbf{A}^{\mathrm{H}} \mathbf{S}\right\}
\end{array}\right] . }
\end{aligned}
$$

From the inverse lemma of the partitioned matrix [21], we know that if

$$
\mathbf{J}_{\boldsymbol{\theta}_{2} \boldsymbol{\theta}_{2}}=\left[\begin{array}{ll}
\mathbf{W}_{11} & \mathbf{W}_{21}^{\mathrm{T}} \\
\mathbf{W}_{21} & \mathbf{W}_{22}
\end{array}\right]
$$

where

$$
\begin{aligned}
& \mathbf{W}_{11}=\operatorname{Re}\left\{\boldsymbol{\Pi}^{\mathrm{H}} \boldsymbol{\Pi}\right\} \\
& \mathbf{W}_{21}=\left[\begin{array}{l}
\operatorname{Re}\left\{\mathbf{A}^{\mathrm{H}} \boldsymbol{\Pi}\right\} \\
\operatorname{Im}\left\{\mathbf{A}^{\mathrm{H}} \boldsymbol{\Pi}\right\}
\end{array}\right] \\
& \mathbf{W}_{22}=\left[\begin{array}{lr}
\operatorname{Re}\left\{\left(\mathbf{A}^{\mathrm{H}} \mathbf{A}\right)\right\} & -\operatorname{Im}\left\{\left(\mathbf{A}^{\mathrm{H}} \mathbf{A}\right)\right\} \\
\operatorname{Im}\left\{\left(\mathbf{A}^{\mathrm{H}} \mathbf{A}\right)\right\} & \operatorname{Re}\left\{\left(\mathbf{A}^{\mathrm{H}} \mathbf{A}\right)\right\}
\end{array}\right] .
\end{aligned}
$$

Then, the inverse of $\mathbf{J}_{\boldsymbol{\theta}_{2} \boldsymbol{\theta}_{2}}$ can be written as

$$
\begin{aligned}
\mathbf{J}_{\boldsymbol{\theta}_{2} \boldsymbol{\theta}_{2}}^{-1}= & {\left[\begin{array}{ll}
\mathbf{0} & \mathbf{0}^{\mathrm{T}} \\
\mathbf{0} & \mathbf{W}_{22}^{-1}
\end{array}\right]+\left[\begin{array}{cc}
\mathbf{I} & v \\
-\mathbf{W}_{22}^{-1} \mathbf{W}_{21} & v
\end{array}\right] } \\
& \times\left(\mathbf{W}_{11}-\mathbf{W}_{21}^{\mathrm{T}} \mathbf{W}_{22}^{-1} \mathbf{W}_{21}\right)^{-1}\left[\mathbf{I},-\mathbf{W}_{21}^{\mathrm{T}} \mathbf{W}_{22}^{-1}\right] .
\end{aligned}
$$

It can be calculated that

$$
\begin{aligned}
& \mathbf{W}_{11}-\mathbf{W}_{21}^{\mathrm{T}} \mathbf{W}_{22}^{-1} \mathbf{W}_{21}=\mathbf{\Upsilon} \\
& \mathbf{W}_{22}^{-1}=\left[\begin{array}{lr}
\operatorname{Re}\left\{\left(\mathbf{A}^{\mathrm{H}} \mathbf{A}\right)^{-1}\right\} & -\operatorname{Im}\left\{\left(\mathbf{A}^{\mathrm{H}} \mathbf{A}\right)^{-1}\right\} \\
\operatorname{Im}\left\{\left(\mathbf{A}^{\mathrm{H}} \mathbf{A}\right)^{-1}\right\} & \operatorname{Re}\left\{\left(\mathbf{A}^{\mathrm{H}} \mathbf{A}\right)^{-1}\right\}
\end{array}\right] \\
& \mathbf{W}_{22}^{-1} \mathbf{W}_{21}=\left[\begin{array}{l}
\operatorname{Re}\left\{\left(\mathbf{A}^{\mathrm{H}} \mathbf{A}\right)^{-1} \mathbf{A}^{\mathrm{H}} \mathbf{\Pi}\right\} \\
\operatorname{Im}\left\{\left(\mathbf{A}^{\mathrm{H}} \mathbf{A}\right)^{-1} \mathbf{A}^{\mathrm{H}} \mathbf{\Pi}\right\}
\end{array}\right] \\
& \mathbf{J} \boldsymbol{\theta}_{1} \boldsymbol{\theta}_{2}\left[\begin{array}{c}
\mathbf{I} \\
-\mathbf{W}_{22}^{-1} \mathbf{W}_{21}
\end{array}\right]=\left[\begin{array}{l}
\operatorname{Re}\{\boldsymbol{\Xi}\} \\
\operatorname{Im}\{\boldsymbol{\Xi}\}
\end{array}\right] \\
& \mathbf{J}_{\boldsymbol{\theta}_{1} \boldsymbol{\theta}_{2}}\left[\begin{array}{cc}
\mathbf{0} & \mathbf{0}^{\mathrm{T}} \\
\mathbf{0} & \mathbf{W}_{22}^{-1}
\end{array}\right] \mathbf{J}_{\boldsymbol{\theta}_{2} \boldsymbol{\theta}_{1}} \\
& =\left[\begin{array}{rr}
\operatorname{Re}\left\{\mathbf{S}^{\mathrm{H}} \mathbf{P}_{\mathbf{A}} \mathbf{S}\right\} & -\operatorname{Im}\left\{\mathbf{S}^{\mathrm{H}} \mathbf{P}_{\mathbf{A}} \mathbf{S}\right\} \\
\operatorname{Im}\left\{\left(\mathbf{S}^{\mathrm{H}} \mathbf{P}_{\mathbf{A}} \mathbf{S}\right\}\right. & \operatorname{Re}\left\{\mathbf{S}^{\mathrm{H}} \mathbf{P}_{\mathbf{A}} \mathbf{S}\right\}
\end{array}\right]
\end{aligned}
$$

where $\mathbf{P}_{\mathbf{A}}=\mathbf{A}\left(\mathbf{A}^{\mathrm{H}} \mathbf{A}\right)^{-1} \mathbf{A}^{\mathrm{H}}$ is the projection matrix onto the subspace spanned by $\mathbf{A}$.
After some algebraic manipulations, the minimal CRB for $\boldsymbol{\theta}_{1}$ can be obtained as

$$
\mathrm{CRB}_{\boldsymbol{\theta}_{1}}=\frac{\sigma^{2}}{2}\left(\hat{\boldsymbol{\Delta}}-\left[\begin{array}{l}
\operatorname{Re}\{\boldsymbol{\Xi}\} \\
\operatorname{Im}\{\boldsymbol{\Xi}\}
\end{array}\right] \mathbf{\Upsilon}^{-1}\left[\operatorname{Re}\{\boldsymbol{\Xi}\}^{\mathrm{T}} \operatorname{Im}\{\boldsymbol{\Xi}\}^{\mathrm{T}}\right]\right)^{\dagger} .
$$

The following equations are useful to derive (62):

$$
\begin{aligned}
\operatorname{Re}\{\mathbf{X}\} \operatorname{Re}\{\mathbf{Y}\}-\operatorname{Im}\{\mathbf{X}\} \operatorname{Im}\{\mathbf{Y}\} & =\operatorname{Re}\{\mathbf{X Y}\} \\
\operatorname{Re}\{\mathbf{X}\} \operatorname{Im}\{\mathbf{Y}\}+\operatorname{Im}\{\mathbf{X}\} \operatorname{Re}\{\mathbf{Y}\} & =\operatorname{Im}\{\mathbf{X Y}\} \\
\operatorname{Re}\left\{\mathbf{X}^{\mathrm{H}}\right\} & =\operatorname{Re}\left\{\mathbf{X}^{\mathrm{T}}\right\} \\
\operatorname{Im}\left\{\mathbf{X}^{\mathrm{H}}\right\} & =-\operatorname{Im}\left\{\mathbf{X}^{\mathrm{T}}\right\} .
\end{aligned}
$$

For more than one data blocks, the $\mathrm{CRB}_{\boldsymbol{\theta}_{1}}$ can be similarly derived as

$\mathrm{CRB}_{\boldsymbol{\theta}_{1}}=\frac{\sigma^{2}}{2}\left(\sum_{N_{k}}\left(\hat{\boldsymbol{\Delta}}-\left[\begin{array}{c}\operatorname{Re}\{\boldsymbol{\Xi}\} \\ \operatorname{Im}\{\boldsymbol{\Xi}\}\end{array}\right] \boldsymbol{\Upsilon}^{-1}\left[\operatorname{Re}\{\boldsymbol{\Xi}\}^{\mathrm{T}} \operatorname{Im}\{\boldsymbol{\Xi}\}^{\mathrm{T}}\right]\right)\right)^{\dagger}$

where $N_{k}$ is the total number of data blocks.

\section{REFERENCES}

[1] D. Falconer, S. L. Ariyavisitakul, A. Benyamin-Seeyar, and B. Eidson, "Frequency domain equalization for single carrier broadband wireless systems," IEEE Commun. Mag., vol. 40, no. 4, pp. 58-66, Apr. 2002.

[2] J. Coon, S. Armour, M. Beach, and J. McGeehan, "Adaptive frequencydomain equalization for single-carrier multiple-input multiple-output wireless transmissions," IEEE Trans. Signal Process., vol. 53, no. 8, pp. 3247-3256, Aug. 2005.

[3] L. Deneire, B. Gyselinckx, and M. Engels, "Training sequence versus cyclic prefix - A new look on single carrier communication," IEEE Commun. Lett., vol. 5, no. 7, pp. 292-294, Jul. 2001.

[4] Y. Zeng and T. S. Ng, "Pilot cyclic prefixed single carrier communication: Channel estimation and equalization," IEEE Signal Process. Lett., vol. 12, no. 1, pp. 56-59, Jan. 2005.

[5] J. A. C. Bingham, "Multicarrier modulation for data transmission: An idea whose time has come," IEEE Commun. Mag., vol. 28, no. 5, pp. 5-14, May 1990.

[6] W. Y. Zou and Y. Wu, "COFDM: An overview," IEEE Trans. Broadcast., vol. 41, no. 1, pp. 1-8, Mar. 1995.

[7] Radio Broadcasting System, Digital Audio Broadcasting (DAB) to Mobile, Portable, and Fixed Receiver, Eur. Telecommun. Stand. Inst., SophiaAntipolis, Valbonne, France, ETS 300 401, 1995-1997.

[8] Digital Broadcasting System Television, Sound, and Data Services; Framing Structure, Channel Coding, and Modulation Digital Terrestrial Television, Eur. Telecommun. Stand. Inst., Sophia-Antipolis, Valbonne, France, ETS 300 744, 1996.

[9] Broadband Radio Access Networks (BRAN): High Performance Radio Local Area Networks (HIPERLAN), Type 2; Systems Overview, Valbonne, France: Eur. Telecommun. Stand. Inst., Sophia-Antipolis, ETR 101683 114, 1999.

[10] Wireless LAN Medium Access Control (MAC) and Physical Layer (PHY) Specifications: High Speed Physical Layer in the $5 \mathrm{GHz}$ Band, IEEE 802.11a, 1999.

[11] E. Moulines, P. Duhamel, J. F. Cardoso, and S. Mayrargue, "Subspace methods for the blind identification of multichannel FIR filters," IEEE Trans. Signal Process., vol. 43, no. 2, pp. 516-525, Feb. 1995.

[12] R. Zhang, "Blind OFDM channel estimation through linear precoding: A subspace approach," in Proc. 36th Asilomar Conf., Pacific Grove, CA, Nov. 2002, vol. 1, pp. 631-633.

[13] C. Li and S. Roy, "Subspace-based blind channel estimation for OFDM by exploiting virtual carriers," IEEE Trans. Wireless Commun., vol. 2, no. 1, pp. 141-150, Jan. 2003.

[14] A. P. Petropulu, R. Zhang, and R. Lin, "Blind OFDM channel estimation through simple linear precoding," IEEE Trans. Wireless Commun., vol. 3 , no. 2, pp. 647-655, Mar. 2004 
[15] M. Moeneclaey, "Synchronization problems in PAM systems," IEEE Trans. Commun., vol. COM-28, no. 8, pp. 1130-1136, Aug. 1980.

[16] S. Fredricsson, "Joint optimization of transmitter and receiver filters in digital PAM systems with a Viterbi detector," IEEE Trans. Inf. Theory, vol. IT-22, no. 2, pp. 200-210, Mar. 1976.

[17] T. Cui and C. Tellambura, "Efficient blind decoding of orthogonal spacetime block codes over time-selective fading channels," in Proc. WCNC, Las Vegas, NV, Apr. 2006, vol. 3, pp. 1644-1649.

[18] X. Cai and A. N. Akansu, "A subspace method for blind channel identification in OFDM systems," in Proc. ICC, New Orleans, LA, Jun. 2000, vol. 2, pp. 929-933.

[19] Y. Zeng and T. S. Ng, "A semi-blind channel estimation method for multiuser multiantenna OFDM systems," IEEE Trans. Signal Process., vol. 52, no. 5, pp. 1419-1429, May 2004.

[20] E. de Carvalho, J. Cioffi, and D. Slock, "Cramér-Rao bounds for blind multichannel estimation," in Proc. IEEE GLOBECOM, San Francisco, CA, Nov. 2000, vol. 2, pp. 1036-1040.

[21] P. Stoica and R. Moses, Introduction to Spectral Analysis. Upper Saddle River, NJ: Prentice-Hall, 1997.

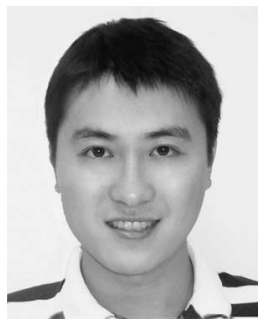

Feifei Gao (S'05) received the B.Eng. degree in information engineering from Xi' an Jiaotong University, Xi' an, Shaanxi, China, in 2002 and the M.Sc. degree from the McMaster University, Hamilton, ON, Canada, in 2004. He is currently working toward the Ph.D. degree at the Department of Electrical Engineering, National University of Singapore, Singapore.

His research interests are in communication theory, broadband wireless communications, signal processing for communications, multiple-inputmultiple-output systems, and array signal processing.

Mr. Gao was the recipient of the President Scholarship from the National University of Singapore.

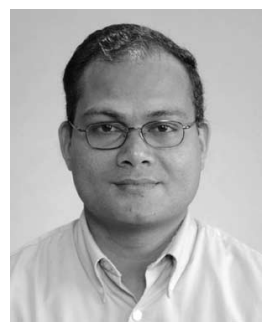

Arumugam Nallanathan (S'97-M'00-SM'05) received the B.Sc. degree (with honors) in electrical engineering from the University of Peradeniya, Peradeniya, Sri Lanka, in 1991, the Certificate in Postgraduate Study in electrical engineering from Cambridge University, Cambridge, U.K., in 1994, and the Ph.D. degree in electrical engineering from the University of Hong Kong, Kowloon, Hong Kong, in 2000 .

Since then, he has been an Assistant Professor with the Department of Electrical and Computer Engineering, National University of Singapore, Singapore. His research interests include high-speed data transmission over wireless links, orthogonal frequencydivision multiplexing, ultrawideband communications systems, and wirelesscommunication theory. He has published more than 90 papers in international journals and conferences.

Dr. Nallanathan serves as an Associate Editor on the Editorial Board of IEEE TRANSACTIONS ON WiRELESS COMMUNICATIONS, IEEE TRANSACTIONS on Vehicular Technology, John Wiley's Wireless Communications and Mobile Computing, and the EURASIP Journal of Wireless Communications and Networking. He also serves as a Guest Editor for the EURASIP Journal of Wireless Communications and Networking: Special issue on UWB Communication Systems-Technology and Applications. He served as a Technical Program Cochair and as a technical program committee member for more than 25 IEEE international conferences.

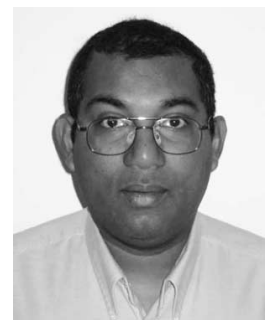

Chintha Tellambura (SM'02) received the B.Sc. degree (with first-class honors) from the University of Moratuwa, Moratuwa, Sri Lanka, in 1986, the M.Sc. degree in electronics from the University of London, London, U.K., in 1988, and the Ph.D. degree in electrical engineering from the University of Victoria, Victoria, BC, Canada, in 1993.

He was a Postdoctoral Research Fellow with the University of Victoria (1993-1994) and the University of Bradford, Bradford, U.K. (1995-1996). He was with Monash University, Melbourne, Australia, from 1997 to 2002. Currently, he is a Professor with the Department of Electrical and Computer Engineering, University of Alberta, Edmonton, AB, Canada. His research interests include coding, communication theory, modulation, equalization, and wireless communications.

Prof. Tellambura is an Associate Editor for the IEEE TRANSACTIONS on Communications and the Area Editor for Wireless Communications Systems and Theory for the IEEE TRANSACTIONS ON WiRELESS COMmUniCATIONS. He was Chair of the Communication Theory Symposium in Globecom'05, St. Louis, MO. 